\title{
First reports of adverse drug reactions
}

(c) Springer Nature Switzerland AG 2020

Table 1 contains an overview of first published case reports of adverse drug reactions and interactions identified in the international literature in recent weeks by Reactions Weekly, the Adis drug safety newsletter. Reactions Weekly provides summaries of adverse drug reaction news sourced from journals, scientific meetings, media releases, regulatory agency websites, and bulletins from the National Centers that participate in the WHO International Drug Monitoring Programme.

Table 1 First reports of adverse drug reactions and interactions

\begin{tabular}{|c|c|}
\hline Drug: adverse reaction & References \\
\hline Bupropion: spontaneous ejaculation & $\begin{array}{l}\text { Uvais NA, et al. Bupropion-induced spontaneous ejaculations. Prim } \\
\text { Care Companion CNS Disord. 2020 2;22(1):19102453. }\end{array}$ \\
\hline $\begin{array}{l}\text { Cabozantinib: atypical posterior reversible encephalopathy syndrome } \\
\text { (serious) }\end{array}$ & $\begin{array}{l}\text { Patwari A, et al. Atypical posterior reversible encephalopathy syndrome } \\
\text { due to oral tyrosine kinase inhibitor cabozantinib: first case report. } \\
\text { Case Rep Oncol 2020;13(2):1013-19 }\end{array}$ \\
\hline $\begin{array}{l}\text { Certolizumab pegol: hypocomplementemic urticarial vasculitis } \\
\text { (serious) }\end{array}$ & $\begin{array}{l}\text { Horai Y, et al. Development of hypocomplementemic urticarial vascu- } \\
\text { litis during certolizumab pegol treatment for rheumatoid arthritis: a } \\
\text { case report. J Clin Pharm Ther. 2020;45(5):1179-92 }\end{array}$ \\
\hline Entecavir: lichenoid drug eruption (serious) & $\begin{array}{l}\text { Cheong XK, et al. "black box warning" rash with entecavir: case report. } \\
\text { BMC Gastroenterol. 2020;20(1):305 }\end{array}$ \\
\hline Methylprednisolone/pyridostigmine: globe subluxation (serious) & $\begin{array}{l}\text { Dam J, et al. Globe subluxation following long-term high-dose } \\
\text { steroid treatment for myasthenia gravis. Case Rep Ophthalmol. } \\
\text { 2020;11(3):534-9 }\end{array}$ \\
\hline Montelukast: diffuse alveolar haemorrhage (serious) & $\begin{array}{l}\text { Martinez Montalvo CM, et al. Montelukast-induced alveolar hem- } \\
\text { orrhage: report of a case [in Spanish]. Acta Medica Peruana. } \\
\text { 2020;37(2):198-203 }\end{array}$ \\
\hline $\begin{array}{l}\text { Natalizumab: measles following measles/mumps/rubella vaccine } \\
\text { (serious) }\end{array}$ & $\begin{array}{l}\text { Miauton A, et al. Vaccine-associated measles in a patient treated with } \\
\text { natalizumab: a case report. BMC Infect Dis. 2020;20(1):75 }\end{array}$ \\
\hline Ocrelizumab: dermatographism & $\begin{array}{l}\text { Raynowska J, et al. Dermatographism associated with ocrelizumab. } \\
\text { Mult Scler Relat Disord. 2020;46:102505 }\end{array}$ \\
\hline Osimertinib: dermatopathic lymphadenopathy (serious) & $\begin{array}{l}\text { Kunimasa K, et al. Dermatopathic lymphadenopathy mimicking } \\
\text { disease progression during osimertinib treatment. J Thorac Oncol. } \\
\text { 2020;15(10):e178-e180 }\end{array}$ \\
\hline $\begin{array}{l}\text { Pantoprazole: drug-induced hypersensitivity in the form of } \\
\text { enterocolitis (serious) }\end{array}$ & $\begin{array}{l}\text { Bouvette G, et al. Drug-induced enterocolitis syndrome (DIES) with } \\
\text { pantoprazole in an adult patient. J Allergy Clin Immunol Pract. } \\
\text { 2020;9(8):3183-4 }\end{array}$ \\
\hline Serenoa repens: fixed-drug eruptions & $\begin{array}{l}\text { Gammoudi R, et al. Fixed drug eruption to Serenoa repens: first case } \\
\text { report and consideration of the use of herbal medicine. Dermatol } \\
\text { Ther. 2020. doi/10.1111/dth. } 14247\end{array}$ \\
\hline Sodium polystyrene sulfonate: rectovaginal fistula (serious) & $\begin{array}{l}\text { Martin LE, et al. Rectovaginal fistula induced by Resonium A: first case } \\
\text { report and literature review. ANZ J Surg. 2019;89(11):E550-E551 }\end{array}$ \\
\hline Ulixertinib: retinopathy (serious) & $\begin{array}{l}\text { Sioufi K, et al. A case of extracellular signal-regulated kinase inhibitor- } \\
\text { associated retinopathy. JAMA Ophthalmol. 2020;138(9):1002-4 }\end{array}$ \\
\hline
\end{tabular}

An event is serious (US FDA MedWatch definition) when the patient outcome is death, life threatening, hospitalization, disability, congenital anomaly or requires intervention to prevent permanent impairment or damage 